\title{
Research on Electric Power EPON Access Network Service Quality Improvement Based On The Intelligent Pipeline Archi- tecture
}

\author{
CHEN Xiaotian ${ }^{1}$ WANG Pan ${ }^{2}$ LIU Shidong ${ }^{3}$ \\ ${ }^{1-3}$ NANJING University of Posts and telecommunications, Nanjing 210003, China
}

\begin{abstract}
Different kinds of applications are carried on EPON-based electric power EPON access network and recently most work is focus on ensuring QoS of important services when congestion happened. Combined those established architectures in public telecommunication networks with characteristics of electric power traffic, a smart-pipe architecture based technical solution and related service-identification algorithm are presented and studied in this paper. Simulation proves that it realizes the on-demand allocation of network resource and improves the intelligent level of the network.
\end{abstract}

Keywords: intelligent pipe; EPON; access network; QoS; service identification

\section{Introduction}

The power company based on EPON technology ${ }^{[1]}$ to construct electric power optical fiber access network covered distribution and comsumption sectors, realizes with including electricity information, community power distribution automated information, data acquisition of electric power applications as well as access of public business system such as household users "three nets fusion" etc ${ }^{[2]}$. In the EPON uplink channel carrying electric power system data, all of the data through different ONU multiplex to one uplink OLT, result in the service quality of important business data is no guarantee in data burst. In view of the above requirements, this paper studies the intelligent pipeline architecture that is suitable for the electrical power EPON access network, puts forward a solution that improve the QoS of important electric power traffic carried on the power EPON access network, by traffic flow sensing technologies such as DPI artificial operation and being control of the user data in order to improve the "intelligence" of the network.

\section{Related Work}

\subsection{Electric Power EPON Access Network}

The power EPON access network can be divided into private and public system, using two sets of communication network and network terminal (OLT) to achieve physical isolation. Electric power private network OLT upward through the optical fiber or directly connected to the 
company's core network, downlink connections community power associated terminal equipment, such as distribution automation equipment, intelligent watthour meter, etc. Public network OLT uplink connection to the Internet, radio and TV network, telecommunication network, downlink households associated terminal equipment.

This article mainly discuss private EPON access network system (the public EPON system is not in the scope of this article). This way of connection exists the following problems:

All of the electric power business system data multiplex on the same link, only through a static configuration port priority way to guarantee the QoS of important system, unable to achieve dynamic on-demand QoS control;

For some specific business (such as video monitoring business in the distribution automation), due to a lack of deep packet inspection (DPI) methods, unable to realize the identification and control of traffic granularity.

\subsection{Intelligent Pipe Solution Of Op- erators}

The intelligent pipeline architecture $^{[3-6]}$, which is top-down divided into application layer, control layer and bearing layer, provides on-demand resources and dynamic demand adjustment. At present several mature intelligent pipeline strategy control technical system including ITU - T RACF (resources and admission control functions) architecture, TISPAN RACS (resources and admission control subsystem) architecture, the BBF BPCF (access network broadband strategy control) architecture and 3GPP PCC (strategy and billing control) architecture [7].

Although the operators about intelligent pipeline have carried out practical application in many aspects, but in the electric field the research about intelligent pipeline is still in the exploratory stage. Power information communication network based on IP technology, with the increase of bearing traffic, these problems on the public network will be emerging in the electric power communication network, this paper carried out the corresponding research from mature architecture network, to solve the power EPON network practical problems.

\section{Power Access Network Intelligent Pipeline Solution Based On Fusion Strategy Control Technology}

\subsection{Power EPON Access Network In- telligent Pipeline Architecture}

Fusion strategy control as the core, draw lessons from mature public intelligent pipeline architecture, the proposed power access network intelligent pipeline architecture is shown in figure 1.

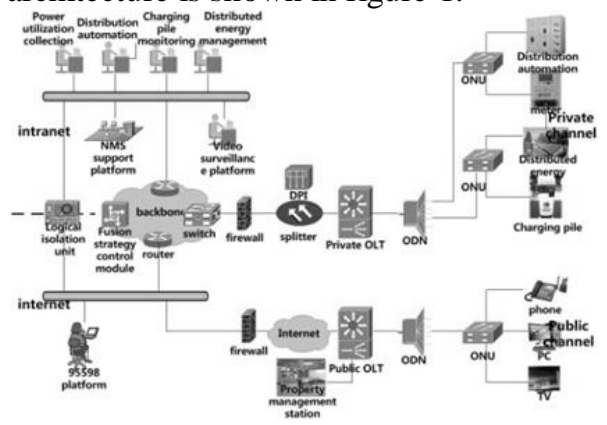

Fig. 1: power EPON access network intelligent pipeline architecture

The architecture is mainly composed of the fusion strategy, policy enforcement, deep packet inspection (DPI), application functions (including application system and application support platform) and EPON access network and IP backbone network QoS control function and so on several parts.

Fusion strategy control module support strategy decision-making and distributed control of EPON access network and IP backbone network. OLT is network awareness and policy enforcement 
points of the EPON access network architecture. OLT used to realize the speed limit and traffic control, or the QoS information transfer to EPON access side to resource scheduling of EPON system, EPON access side shall be carried out in accordance with the QoS parameters of the distributed bandwidth resource scheduling and management. OLT and backbone network equipment (backbone policy enforcement point) joint guarantee end-to-end QoS of service. DPI module in parallel or in series way access to the Uplink of OLT, it use the DPI technology identification and reporting of the service, determine the category of the service, to provide different levels of network services.

\subsection{Power EPON Access Network In- telligent Pipeline Solution}

The QoS improvement solutions of EPON access network based on the framework of the previous section can be broken into 3 phases :Strategy Generation Phase, strategy distribution stage and strategy implementation stage. In phase 1, Policy control module according to various information from different channels carries out summary decision-making, to generate control strategy. Strategy control module distributing such as QoS control strategy to policy enforcement point in the EPON access network and backbone network, dynamic control of behavior of the execution point. Policy enforcement points mapped distributed control strategy to the QoS parameters, and these parameters are passed to related parts of the EPON access network. EPON equipment according to these parameters mapped to resource scheduling priority, so as to ensure the QoS of the application.

\section{Research On Video Monitoring Application Identification Model And Corresponding Algorithm Based On SAT}

In this paper, based on the architecture of section 3, recognition of video surveillance and other video services is studied, service recognition model and algorithm based on session affinity technology (SAT) is verified.

\subsection{Research Of Video Monitoring Application Identification Model Based On DPI Technology}

Video surveillance traffic ${ }^{[8]}$ and other distribution automation application, electricity information gathering are multiplexed on OLT uplink for private network. Because the control message used to access the real-time video service (based on SIP) and subsequent video session data (based on RTP) in the data frame header and other electric business data is no difference, so the simple service identification method based on the port and MAC address cannot distinguish.

In this paper, based on the intelligent pipeline architecture, using DPI technology, design a service identification model suitable for video monitoring business as shown in figure 2. The whole model down-top divide into data collecting layer, protocol analysis layer, application identification layer and application process layer.

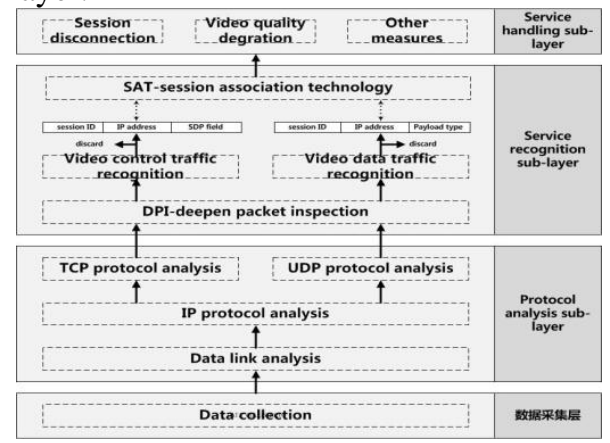

Fig. 2: the video service identification model based on DPI 
Application Identification Layer is the core of the whole model, it is based on the protocol analysis provided by IP header information, a transport layer segment information and packet payload, identify service through DPI technology.

According to the analysis information of service recognition layer, and combined with other information, policy control module generates a processing strategy on a particular video business: demolitions the video session or degradation video compression format to alleviate the load of network bandwidth.

\subsection{The Video Monitoring Service Recognition Algorithm Based On SAT}

Video session control service recognition module is mainly through to the interaction message analysis in the process of the video session setting up and dismantling, establish the corresponding video session information table, the table is used to store all of the currently valid video session information. Include the session ID, IP address, terminal capability information, as well as the session parameter, etc. The table is associated with the corresponding session data traffic through session ID, IP address and other key words. When need to deal with the corresponding application, this kind of relationship, can help to simplify the operation process.

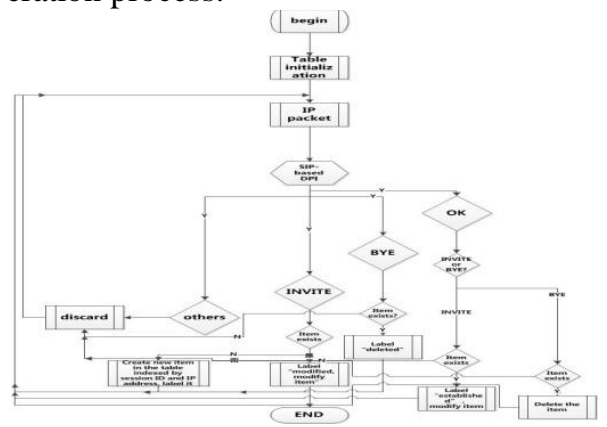

Fig. 3: video session control application message identification algorithm
The recognition algorithm of video session control message as shown in figure 3. Mainly three types of video session control message processing: INVITE, $\mathrm{BYE}$ and $\mathrm{OK}$.

After the construction of the video session, the transmission of video service stream data between endpoints through RTP protocol. Video session data traffic identification module based on RTP protocol for the video stream data analysis, only belongs to the video session information in the table an activation of the video (status to "build") traffic data flow information is stored. Stored information includes the RTP payload information, MAC address information and the same endpoint of the video traffic recently to link usage statistics.

Due to in front the video traffic converge a plurality of terminal data, so a single IP address cannot be a one-to-one relationship, in the DPI analysis of video traffic flow data, using the session Association Technology based on IP address and session ID to ensure that only, the flow without a data connectivity through the SAT association to the established video session. This correlation can provide the necessary information for policy control module by using the video session related information in the table (such as the terminal capability information).

Based on DPI analysis for video traffic information and other service information, strategy control module can generate the corresponding control strategies: when the OLT uplink congestion, can choose video of low level take forcible demolition, to renegotiate to lowquality format video of lower bandwidth, transmission delay, etc to ensure reliable, timely delivery of high priority service. Control strategy is sent to the source, the destination and policy enforcement point in the network, mapped as flow control, port priority setting, queue scheduling and other specific QoS control method. 


\section{Project Feasibility}

Figure 4 the system host and several IP cameras with different ONU to reuse the same uplink OLT. Between system host and application test system to simulate higher priority applications, and data interaction between IP camera and video monitoring platform is DPI object, represents the QoS level lower applications, when the uplink congestion occurs control will be necessary to ensure the normal conduct of important business.

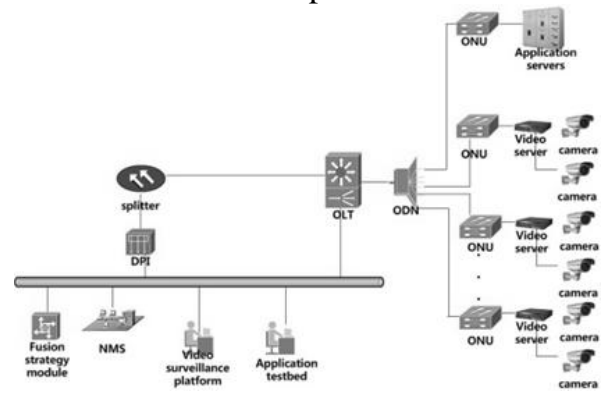

Fig. 4: experimental network

The experiment through the port rate limiting feature set OLT uplink port speed is 50Mbps. Through FTP simulate important applications, video surveillance platform gradually increase the number of channels of the camera to produce module congestion. In the initial conditions the rate of FTP to $1 \mathrm{Mbps}$, the camera video formats to $720 \mathrm{P}, 25 \mathrm{~F} / \mathrm{s}$, bandwidth occupied by single video stream is about $3.5 \mathrm{Mbps}$.

In the absence of enable fusion strategy control module, when the video number increased to a certain number (13-14), the FTP rate decreased obviously, and the portion of the video stream appears mosaic phenomenon.

After fusion strategy control module enabled by video format degraded to ensure link bandwidth, when the video channels to the same number, rate of FTP changed little, still maintained at about $1 \mathrm{Mbps}$. Most of the video stream to maintain the original quality, a video stream quality is degraded to 4CIF/D1
(25F/s, 1.5Mbps bandwidth). Analysis of control packets in the experiment showed: video server of video surveillance platform and the camera for the renegotiation, select low-quality image transmission that quality is low but bandwidth take up less. And OLT automatically to this ONU occupy the uplink bandwidth of the video is compressed.

\section{Conclusion}

Combined with the characteristics of electric power information network and the network reference architecture proposed an technical scheme improved QoS of power EPON access network and the corresponding service recognition algorithm. Experimental results show that, due to the introduction of service identification model based on DPI technology, which can realize service granularity ondemand control based on intelligent pipeline architecture, to ensure the service quality of key service.

\section{References}

[1] Zhang P,Yan K. FTTx PON technology and applications[M]. Beijing: The People's Posts and Telecommunications Press, 2009.

[2] State Grid manufacturer's standard Q/GDW 541-2010. Typical design for PFTTH, 2010.

[3] 3GPP TS 23.203. Policy and charging control architecture; (release 7), 2008.

[4] ETSI ES 282 003. Resource and admission control sub-system (RACS); functional architecture; (release 2), 2008.

[5] ITU-T draft recommendation Y.RACF Release 2 Version 0.4.0, 2007. 3GPP TR 23.839 V0.2.0. Study on support of BBF access interworking, 2010.

[6] Zhao H. L,Xu X,Chen Y.Q. Study on wise pipeline[J]. Telecommunications Science:2011,(3):7-11.

[7] Zhang J.P,Li H. Architecture and technologies for telecommunication network[M]. BJ: Electronic Industry 
Press, 2012.

[8] State Grid manufacturer's standard Q/GDW 517.1-2010. Grid video surveillance system and interfaces session 1 technical requirements, 2010 . 